Revue d'histoire de l'Amérique française

PEVUE D.HISTOIRE DE L'AMÉRIQUE FRANÇAISE

\title{
Quelques officiers de Montcalm (suite)
}

\section{Aegidius Fauteux}

Volume 5, numéro 3, décembre 1951

URI : https://id.erudit.org/iderudit/801719ar

DOI : https://doi.org/10.7202/801719ar

Aller au sommaire du numéro

Éditeur(s)

Institut d'histoire de l'Amérique française

ISSN

0035-2357 (imprimé)

1492-1383 (numérique)

Découvrir la revue

Citer ce document

Fauteux, A. (1951). Quelques officiers de Montcalm (suite). Revue d'histoire de l'Amérique française, 5(3), 404-415. https://doi.org/10.7202/801719ar d'utilisation que vous pouvez consulter en ligne.

https://apropos.erudit.org/fr/usagers/politique-dutilisation/ 


\section{QUELQUES OFFICIERS DE MONTCALM*}

Extrait d'un ouvrage manuscrit d'Aegidius Fauteux, intitulé: "Les Officiers de Montcalm".

BEAUCLAIR (Joseph de)

Capitaine dans le 2e bataillon de la Sarre, il s'embarqua à Brest en mars 1756, en même temps que le marquis de Montcalm.

Dès le commencement de 1758, il faillit laisser ses os en Canada. En effet, le 10 mars de cette année, Montcalm écrit de Montréal à Bourlamaque: Nous avons été au moment de perdre M. de Beauclair; ... inflammation au bas ventre, poitrine affectée et on n'osait le saigner".

Il se rétablit assez tôt cependant pour prendre part, le 8 juillet 1758, à la bataille de Carillon où il fut blessé. Le 20 octobre suivant, il reçoit une gratification de $\mathbf{4 0 0}$ livres sur le quatrième denier.

Dans son mémoire de recommandation de la fin de 1759 , le chevalier de Lévis sollicite pour lui une augmentation de pension et ajoute: "Cet officier sert avec tout le zèle et application possibles; il a commandé une compagnie de volontaires pendant la campagne dernière et s'est acquitté avec distinction de toutes les commissions dont il a été chargé".

Le 30 octobre 1757, le chevalier de Lévis l'avait déjà recommandé en ces termes au maréchal de Belle-Isle:

Permettez-moi d'avoir l'honneur de vous recommander le Sieur de Beauclair, capitaine au régiment de la Sarre, et de me joindre à M. le Marquis de Montcalm pour vous supplier de lui accorder la grâce qu'il vous demande, qui est de lui rendre reversible une pension de 300 livres que $M$. le Chevalier

* Voir, Revue d'Histoire de l'Amérique française, III, no 3: 367-82; IV, no 4: 521-529. 
de Beauclair, son oncle, avait, dont il a appris la mort cette année. Il était maréchal des logis de la première compagnie de mousquetaires. J'ose vous assurer que le Sieur de Beauclair mérite votre protection; il n'a pour fortune qu'une légitime d'Auvergne; c'est un officier de distinction; il a eté blessé considérablement à la tête à notre affaire du 8 juillet et sa blessure n'est point encore fermée...

Cette recomandation ne fut pas tout à fait sans effet, car le 12 février 1760, le sieur de Beauclair obtenait une pension de 200 livres faisant partie de celle dont jouissait son oncle.

En août 1759, il était détaché de Québec pendant le siège pour aller se mettre aux ordres du chevalier de Lévis, qui dirigeait les opérations du côté d'en haut. Le 29 de ce mois, Lévis qui se trouvait à l'île Orakouinton ou Fort Lévis, notait dans son Journal l'arrivée de cent hommes de troupes de terre commandés par M. Beauclair et de cent miliciens. Il s'agit sans doute du détachement dont parle Malartic comme étant parti de Québec le 11 août et composé de cent volontaires des troupes de terre. Dans le volume de la Collection des manuscrits de Lévis intitulé Lettres de quelques particuliers, on trouve plusieurs lettres écrites par Beauclair à Lévis, du 7 septembre au 31 octobre 1759. Au fort Lévis où il commandait, il n'avait guère qu'à surveiller des travaux de fortifications et cette inaction lui pesait. A plusieurs reprises, il s'en plaint au chevalier de Lévis.

Vous aviez cru, lui écrit-il, le 23 octobre, en me faisant partir de Québec, m'employer à la guerre; je ne demandais que cela pour vous témoigner mon zèle à remplir ce dont vous me chargiez; mais ayant joui ici de la plus grande tranquillité, tandis que notre armée avait tous les jours l'ennemi sur les bras, j'oserais vous prier, au cas qu'il y eût dans l'hiver quelque parti, de vouloir m'employer. Il m'importe fort peu d'être premier ou second.

Quelques jours plus tard il revient à la charge:

Je ne cesserai point de prier de m'employer en hiver, s'il y a quelque détachement en campagne. Il ne faudrait pas qu'il fut dit qu'un commandant de volontaires n'a fait que piocher la terre tandis que toutes les troupes de l'armée de Québec ont été écrasées. 
Au commencement de novembre 1759 , il fut enfin relevé du commandement du Fort Lévis par Desandrouins et il put repartir pour Québec, mais il avait manqué la bataille des Plaines d'Abraham. Présent à la bataille de Sainte-Foy, le 28 avril 1760, il y fut dangereusement blessé d'un coup de feu au bras. Il resta prisonnier à l'Hôpital général jusqu'au départ pour la France en octobre suivant. Le 10 février 1761, le roi le fait chevalier de Saint-Louis, mais M. de Lévis n'en sollicite pas moins pour lui, quelques mois plus tard, une nouvelle faveur sous forme d'augmentation de pension:

Deux cent livres, dit-il, lui ont été accordées à la mort d'un de ses oncles; il a reçu depuis deux blessures considérables et s'est fort distingué à l'affaire du 28 avril 1760 où il a combattu jusqu'à la fin le l'action quoiqu'il eût un bras cassé dès le commencement. La croix de Saint-Louis vient de lui être accordee comme ancien. C'est un homme de condition pauvre, dont les talents méritent récompense. Il a commandé les volontaires dans plusieurs occasions.

Le registre du régiment de la Sarre donne comme suit l'état de ces services:

Né le 16 août 1728; est d'Aurillac. Lieutenant le 15 juin 1747; devenu enseigne à la réforme le 18 mars 1749; lieutenant en second de grenadiers le 2 octobre 1750; remplacé lieutenant le 30 avril 1751; capitaine le 22 mars 1756; rang de colonel le 9 juillet 1762; réformé à la nouvelle composition de 1763; remplacé à une compagnie le 5 décembre 1763; lieutenantcolonel du régiment de Vexin, le 27 novembre 1765. Blessé d'un coup de feu à la bataille devant Québec, $\in \mathbf{n} 1760$.

Note de 1764: A des connaissances et de l'ambition et le grade de colonel pour avoir été l'aide-de-camp de M. de Lévis en Canada.

Il y avait en même temps dans le régiment de la Sarre, en 1763 et après, deux capitaines de Beauclair, natifs tous les deux d'Aurillac. L'un, notre capitaine du Canada, était connu sous le nom de Beauclair; l'autre était appelé Messac, mais dans le registre du régiment on le désigne plus au long Gérard de Beauclair de Messac. Dans son Impôt du sang, d'Hozier enregistre Gérard de Beaucaire (sic) de Messac, chevalier de Saint-Louis, capitaine de grenadiers au régi- 
ment de la Sarre, blessé en 1758 à l'affaire de Carion et retiré du service en 1779. Il a confondu les deux frères; Jos. de Beauclair qui fut blessé à Carillon n'a pas été capitaine de grenadiers, et Gérard de Beauclair de Messac n'est jamais venu en Canada. Les deux capitaines de la Sarre étaient fils de Jean-Baptiste de Beauclair, chev. Sgr. de Messac et de Marguerite-Claude de Boschatel.

Le Sr de Beauclair n'a pas trop souffert dans son nom aux mains des éditeurs ou imprimeurs. L'on ne rencontre guère que de légères variations: Beauclair ou Bauclair. Il n'y a que Danré de Blanzy qui, dans l'acte de dépôt du testament de M. de Senezergues, le 5 janvier 1760, l'appelle Joseph de Boisclair. Mais l'officier luimême signe comme témoin au bas de l'acte: Beauclair. L'abbé Tanguay a commis une pire offense envers son régiment lorsqu'il parle (II: 169) de M. Beauclair, capitaine au régiment de Lazare!

Montcalm, dans une de ses lettres à Bourlamaque (p. 279), parle d'un capitaine Beaudoin de la Sarre qui est assurément le capitaine Beauclair, mais l'erreur ne peut être attribuée qu'à une mauvaise lecture du manuscrit original par l'abbé Casgrain.

La famille de Beauclair, d'ancienne noblesse d'Auvergne, a eu pour berceau le château de son nom situé sur le territoire de la commune actuelle de Fontanges, dans le canton de Salers. Elle a été maintenue dans sa noblesse en 1666 par l'intendant Fortia, après avoir prouvé sa filiation depuis 1415 .

Armes: d'or à 3 chevrons de gueules; au chef d'argent chargé de cinq mouchetures de sable.

\section{SOURCES:}

Lettres de la Cour de Versailles, 130, 216, 227. - Lettres du Chevalier de Lévis, 216, 459. - Journal des campagnes de Lévis, 200. Lettres et pièces militaires, 305. - Lettres de Bourlamaque, 81, 209, 297. - Lettres de Montcalm, 149, 222, 225. - Lettres de quelques particuliers, 44, 49, 50, 51, 86, 98, 105, 177-194 et 209. - Tanguay, $A$ travers les registres, 177 . - $\mathrm{BRH}, \mathrm{XX}: 371$. - Registre du régiment de La Sarre. - Malartic, Campagne au Canada, 269. D'Hozier, Impót du sang, I: 144. - Chaix d'Est-Ange, Dictionnaire des familles françaises, III: 140. 


\section{BLANCHARD (Louis-René) Sieur du Val}

Etait lieutenant de la compagnie de Matissart dans le 2e bataillon de Languedoc, lorsqu'il s'embarqua à Brest, à bord de l'Actif, le 6 avril 1755. Présent à la bataille de Carillon, le 8 juillet 1758, il y fut blessé. Le 25 juillet suivant, il fut nommé pour exploiter la compagnie de Dutertre, prisonnier, à la place du Sr Dupuy, mort. Dans l'état des grâces accordées le 20 octobre 1758 , on le trouve porté pour une gratification de 200 livres sur le quatrième denier. Le 12 février 1760, il recevait, comme capitaine, une nouvelle gratification de 400 livres.

Le registre du régiment de Languedoc donne comme suit ses états de services:

Louis Duval de Blanchard, né en 1730 à Hennebont (Bretagne). Lieutenant dans Languedoc le 1er septembre 1747; réformé en 1749; lieutenant dans la milice de Bretagne le 25 février 1750; lieutenant dans Languedoc le 1 er avril 1755; capitaine le 25 juillet 1758; réformé en 1763; remplacé capitaine le ler janvier 1766 . Blessé à l'attaque du Borgo en Corse de trois coups de fusil, le 8 aout 1768; a commandé une compagnie de volontaires en Corse pendant la campagne de 1769. - Chevalier de Saint-Louis le 5 mai 1772.

Note de 1763: - D'une très grande valeur, sert avec distinction à la guerre.

Note de 1776: - Excellent officier à la guerre, M. le Cte de Marbeuf l'a presque toujours employé en corps.

D'après l'État des pensions, il reçut en 1769 une pension de 400 livres sur les fonds de la guerre "en considération de la distinction avec laquelle il s'était comporté dans les combats livrés dans l'île de la Corse, les 7 et 8 mai 1769".

M. de Milleville, dans son Armorial historique, nous apprend que Louis-René de Blanchard, seigneur du Val de Kerquer, capitaine dans Languedoc, fut nommé en 1780 au commandement de la ville de Saint-Florent, en Corse et y mourut le 6 juin 1786 .

Le 7 janvier 1760, il avait épousé aux Trois-Rivières, Charlotte, fille de Nicolas-Joseph de Noyelles, lieutenant du roi aux TroisRivières et de Charlotte Petit de Livilliers. Lui-même est dit à l'acte fils de Mathurin Blanchard Duval et de Françoise Boutouillié, de Hennebont, en Basse-Bretagne. 
Nous lui connaissons quatre enfants:

1. Marie-Louise, qui a da naitre en 1761 car sur l'Etat des pensions de 1789 , où elle figure pour 150 livres depuis 1787, elle est dite âgée de 28 ans.

2. Marguerite-Félicité, baptisée à Loches (paroisse de St-Ours) le 23 novembre 1769 et qui, après avoir été présentée à l'École royale de Saint-Cyr, le 18 mars 1778, en sortit le 11 novembre 1789 , recevant sa dot le 10 avril 1790 .

3. Françoise, qui a dû naître en 1772 , car dans l'État des pensions de 1789 où elle figure pour une pension de 150 livres depuis 1787, elle est dite âgée de 17 ans.

4. Jean-Baptiste-Noël de Blanchard du Val, né le 21 décembre 1775, et mort à 72 ans au château du Val en Morbihan, le 24 aout 1846. Il était chevalier de St-Louis, en même temps qu'officier de la Légion d'honneur et que chevalier de l'Ordre de Saint-Ferdinand d'Espagne. Il avait épousé en 1801 Marie-Jeanne-Sophie, fille de Messire Alexandre-Toussaint Briant, seigneur de la Villeneuve, capitaine de grenadiers au régiment de Barrois et chevalier de SaintLouis. De ce mariage sont issus:

a) Ange-Vincent-Arsace, né le 17 février 1808 et qui en 1845 était capitaine de voltigeurs au 51e régiment de ligne.

b) Marie-Thérèse-Sabine.

c) Marie-Elodie.

La famille Blanchard appartient à l'ancienne noblesse de Bretagne. La branche aînée est dite de la Buharaye. Quant à la seconde, celle du Val, elle fut longtemps dans une situation modeste. Noble homme Gilles Blanchard de Kerpunce, fils de Guy, marié à Jeanne Gontier, fut reçu en 1641 notaire, priseur et avocat des juridictions inférieures d'Hennebout, puis en 1642 sénéchal de Sainte-Geneviève, racheta en 1650 les droits de son frère aîné, Jean Blanchard de Kercaizre sur la terre du Val, vendit en 1666 son étude pour se faire négociant et n'en fut pas moins maintenu dans sa noblesse par arrêt du 10 juillet 1669. Il fut l'aïeul de notre capitaine du Languedoc.

Armes: d'azur à trois croissants d'argent, 2 en chef, 1 en pointe. 


\section{SOURCES:}

Lettres de la Cour de Versailles, 131, 151, 213. - Lettres et pièces militaires, 291. - Etat des pensions, III: 89. - Tanguay, II: 307. - Registre du régiment de Languedoc. - Chaix d'EstAnge, Dict. des familles françaises, IV: 345, 347. - Fleury-Vindry, Les demoiselles de Saint-Cyr, 59. - D'Hozier, L'Impôt du sang, I: 216. - Henry de Milleville, Armorial historique, 54.

BONAFOUS (Le sieur)

Officier d'artillerie, il est nommé en Canada le 19 mars 1757, enseigne en second de la première compagnie de canonniers-bombardiers, commandée par Jacau de Fiedmont. En octobre 1758, il était déjà passé lieutenant, mais nous n'avons pu découvrir la date de sa promotion. A cette date, il reçoit comme lieutenant d'artillerie, une gratification de 400 livres sur le 4 e denier.

A la fin de 1759, dans un mémoire des grâces demandées, on trouve à côté de son nom, l'apostille suivante du chev. de Lévis:

"Bonafous, lieutenant en premier. - Commission de capitaine. Sert dans Royal-Artillerie de 1746, passé en Canada en 1757, employé toujours dans les forts et pris prisonnier à Niagara où il a servi avec distinction, ayant été blessé plusieurs fois le même jour".

En marge on lit: Commission de capitaine, mais la promotion promise n'eut pas lieu, car, deux fois encore en 1760 , Lévis la redemande et même en 1761 le Sr Bonafous l'attendait encore. En décembre 1760 , le chevalier de Lévis accole à sa demande l'apostille suivante:

"Bonefous - Cet officier sert depuis longtemps; être prisonnier dont il n'est pas encore de retour l'a privé d'avoir des grâces; a très bien servi partout où il s'est trouvé".

En 1761, le Sr Bonafous a présenté lui-même à M. de Cremille, le mémoire suivant qui contient ses états de services.

"Le Sr Bonafous de Caminel a commencé à servir le ler février 1746, en qualité de cadet dans Royal-Artillerie, bataillon de Fontenay. A fait en cette qualité le siège de Mons, Charleroy, Namur; a été à la bataille de Rancoux et Lawfelt. Il a été fait sous-lieutenant le 1er août 1747; a fait en cette qualité le siège de Maestricht. Il est 
parvenu à être lieutenant en premier en continuant au dit corps jusques au ler mars 1757. Dans ce temps, a été détaché pour servir à la suite de l'artillerie de Marine en Canada où il a fait les campagnes de 1757,1758 et partie de 1759 .

"Pendant le cours de ce temps il lui a été confié la direction des fortifications de Québec, le commandement et approvisionnement de l'artillerie des forts de Carillon, la Pointe à la Chevelure et Niagara.

"Dans toutes les parties où il a été employé, il a donné des preuves de son zèle pour le Service de Sa Majesté et a eu occasion de le prouver plus particulièrement au siège de Niagara où il était le seul officier d'artillerie, et, par conséquent, obligé de passer 17 jours et 17 nuits sur les remparts. De plus, il a fait les fonctions d'ingénieur et rempli l'un et l'autre service avec distinction, de l'aveu de toute la garnison et attestation du Commandant. Il a reçu dans le cours de ce siège 3 blessures, à savoir le 17 juillet 1759, un éclat de bombe à la tête; le $19 \mathrm{du}$ dit, un autre éclat de bombe à la tête avec grande contusion, et qui lui a laissé de fréquents maux de tête et affaibli l'ouie de l'oreille droite; le $21 \mathrm{du}$ dit, il a été atteint d'un biscayen, sur la deuxième et troisième des fausses côtes, avec grande contusion. Quoique ses blessures le fissent extraordinairement souffrir, il n'a jamais quitté son poste. Si le Sr Bonafous n'a pas continué les campagnes de 1759 et 1760 , c'est qu'à la reddition du fort, il a été conduit prisonnier de guerre à la Nouvelle Angleterre et détenu 18 mois malgré les représentations qui ont été faites pour l'exécution du cartel. Cette décision a été contraire à l'avancement $\mathrm{du}$ dit Sr Bonafous et lui a causé la perte de tous ses équipages qu'il a été obligé de laisser à Québec et qui lui ont été pillés lors du siège de cette place.

"Le Sr de Bonafous vous supplie, Monseigneur, d'avoir égard à ses services et de lui faire accorder la commission de capitaine et la croix de St-Louis, l'un et l'autre brevet de la même date qu'il a plu à Sa Majesté de l'accorder au sieur Duverny".

Et à ce mémoire le chevalier de Lévis lui-même ajoute la recommandation suivante en date du 24 mars 1761:

"Je prie Monsieur de Cremille de vouloir bien avoir égard au mémoire du Sr de Bonafous; il est dans l'exacte vérité. C'est un officier de grand mérite et de grande distinction. Je ne l'ai pas com- 
pris dans les mémoires des grâces que j'ai demandées parce qu'on le croyait mort dans la Nouvelle Angleterre où il était détenu prisonnier".

Nous avons déjà vu que, malgré ce qui précède, le chevalier avait déjà compris le Sr Bonafous dans deux mémoires de grâces demandées en 1759 et en 1760 , mais là où le défaut de mémoire du général apparaît le plus singulier c'est lorsqu'il dit qu'il croyait le Sr Bonafous mort dans la Nouvelle Angleterre. Le 14 juin 1760, le chev. de Lévis écrit lui-même au général Amherst qu'il lui envoie le Sr de Bonneau de Guyenne pour régler l'affaire des prisonniers et que le dit $\mathrm{Sr}$ de Bonneau est autorisé par le marquis de Vaudreuil a traiter l'échange du Sr de Bonafous, lieutenant d'artillerie, dont l'avancement est malheureusement retardé par sa détention. Quoique le général eât répondu à Lévis dès le 26 juin: "J'accorde volontiers l'échange de MM. de Bonafous et de la Miltière", le Sr de Bonafous ne paraît avoir été libéré que beaucoup plus tard. Ce n'est en effet que le 9 mars 1761 que le Sr de Bonneau écrit du Havre de Grâce au duc de Choiseul, qu'il arrive de la Nouvelle Angleterre avec MM. de Bonafous et de la Miltière qui ont été échangés.

Dans son mémoire à $M$. de Cremille, notre officier d'artillerie se nomme lui-même Bonafous de Caminel. Il a dâ emprunter ce surnom au bourg de Caminel dans le département du Lot et cela indiquerait qu'il était de l'Agénois.

On a imprimé de toutes façons le nom de $\mathrm{Sr}$ de Bonafous, Bunnefonds, Bounnaffons et jusqu'à Bouraffons. C'est cependant Bonnefonds qu'il a été le plus souvent appelé, quoique à tort et par pure fantaisie orthographique.

Chacun sait que depuis 1887 on s'obstine a le considérer sous ce nom de Bonnefons, comme l'auteur du Voyage au Canada, de 1751 à 1761 publié par l'abbé Casgrain et dont le manuscrit n'est signé que des initiales J.C.B. Pour les deux seules raisons que l'auteur avait été quelque temps avec Pouchot au fort Niagara et que son nom devait commencer par la lettre B, l'abbé Casgrain a conclu un peu rapidement qu'il devait être Bonafous, ou, comme il l'appelait, Bonnefonds, et tout le monde a suivi. Après ce qui vient d'être dit de Bonafous d'après les documents officiels, peut-être finira-t-on par comprendre qu'il n'y a rien de commun entre l'officier d'artillerie arrivé en 1757 et le simple soldat, passé plus tard commis, qui a 
raconté son séjour en Canada de 1751 à 1761 . La question a été déjà étudiée dans le Bulletin des Recherches historiques de mars 1931.

\section{SOURCES:}

Lettres du chev. de Lévis, 344, 352, 426, 439. - Lettres de la Cour de Versailles, 133. - Collection de Manuscrits, IV: 307. - Rapp. des Archives de Québec (1923-1924), 41. - Knox, Historical Journal, II: 457; III: 187, 244, 297. - O'Callaghan, X: 977, 992. - Rapp. des Archives du Canada (1905), I: 233, 282. - Lettres de Bourlamaque, 221. - Lettres et pièces militaires, 19. - Bulletin des Recherches historiques, (XXXVII), 163. - Pouchot, Mémoires, II: passim.

BOUCHEL D'ORCEVAL (Charles-Roch de)

Né à Québec, le 27 août 1740, il était le fils de Jacques-François Bouchel d'Orceval, ancien mousquetaire, lieutenant de cavalerie et avocat au parlement de Paris qui, en 1731, fut transféré par lettre de cachet de la prison du Château de Guise au Canada. Fils luimême de J.-B. Bouchel d'Orceval, lieutenant-général des eaux et forêts du duché de Valois, ce fils de famille déporté, épousa à Québec en 1740 Françøise Cardinet, il en eut plusieurs enfants, dont une fille Agnès, qui épousa plus tard en France François-Antoine Jarry de Mancy, mestre de camp de cavalerie et chevalier de St-Louis, et Roch-Charles, le sujet de la présente notice.

Roch-Charles passa en France en 1752. Le 26 février de cette année le ministre écrit à $\mathrm{M}$. Bigot de donner passage sur la Seine au Sr Dorceval, âgé de 11 à 12 ans, à qui son oncle, l'abbé Dorceval, chanoine de Noyon, désire procurer une éducation convenable. Le jeune homme revint au Canada en 1756, après un séjour d'environ 4 ans en France. C'est en effet le 26 mars 1756 que le ministre annonce au duc de Gesvres qu'il a procuré au Sr Dorceval le passage en Canada demandé pour lui. Quelques mois après son arrivée, le 4 novembre 1757, il est nommé lieutenant en second de la compagnie de la Bresne dans le régiment du Berry, à la place de Jacques Theron promu lieutenant. Dans le mémoire de proposition le marquis de Montcalm note: "C'est un homme de condition et qui est très proche parent de $M$. le Maréchal de Conflans. Il était cadet dans le 
régiment de Béarn où il a fait deux campagnes en cette qualité. On le propose pour Berry parce qu'il n'y a point d'emploi dans Béarn".

La parenté du Sr d'Orceval avec le maréchal de Conflans s'explique comme suit: Hubert de Conflans, maréchal de France, né en 1690 et mort en 1777, était le fils de Robert-Aimé de Conflans et de Anne-Charlotte du Bouchel, sœur aînée de J.-B. Bouchel d'Orceval et, par conséquent grand'tante paternelle de Roch-Charles d'Orceval.

Le 25 juillet 1758, le Sr d'Orceval est transféré de Berry dans Béarn où il est nommé à la lieutenance de la Cie de Trepezec vacante par la démission du Sr de Rosmorduc. En janvier 1760, il abandonne à son tour pour passer en France et il est remplacé par le Sr de la Tessonière à la lieutenance de la compagnie de Figuiery, ancienne compagnie Trepezec.

Dans une lettre à Bourlamaque que l'abbé Casgrain a datée du 5 novembre 1758 , mais qui doit être de 1757 , on rencontre une piquante appréciation du marquis de Montcalm sur le compte du jeune d'Orceval qui, quoique officier, n'avait que 17 ans. "Vous vous intéressez, monsieur, ainsi que moi à $M$. d'Arseval (sic) qui est un vrai panier percé; grondez-le bien, accoutumez-le à vivre de peu. MM. de Berry ont promis de l'établir dans quelque quartier, qu'il y passe son hiver sans faire aucune dépense. Voyez de convenir avec Surimeau de ce qu'on lui donnera, par mois, pour appointements, dont la retenue sera faite à l'arrivée de ses lettres. Il s'est avisé de prêter, à ce qu'on m'a dit, 160 livres au petit Lanaudière. Je l'en ai bien grondé, comme M. Gaillard qui s'avise de lui vendre une montre à crédit à qui il doit 310 livres. Voyez que le petit LaNaudière ne remette point l'argent à $M$. d'Arseval, mais faites-le remettre à $M$. Gaillard, à compte; parlez-en à la mère. Au reste, le moindre mécontentement que vous puissiez avoir de M. d'Arseval, mettez-le moi en prison; c'est un vrai service que vous lui rendrez et dont il vous aura obligation, un jour, et a moi".

A partir de son départ, en France, vers janvier 1760, nous perdons la trace de Charles-Roch d'Orceval.

La famille de Bouchel, originaire de Picardie, et dont la branche d'Orceval s'est établie dans le Valois vers la fin du XVIe siècle, blasonne comme suit:

D'azur semé de trefle d'argent au lion passant, aussi d'argent, brochant sur le tout. 


\section{SOURCES:}

Lettres de la Cour de Versailles, 157, 160. - Lettres du chev. de Lévis, 406. - Lettres de Bourlamaque, 270, 325. - LachesnayeDesbois, 3e éd., III: 640-641. - Roy et Massicotte, Armorial II: 51. - Tanguay, Dictionnaire, II: 373. - Bulletin des Recherches historiques, XIV: 186, 187; XXXVI: 257.

Aegidius Fauteux

\section{LES É T U DES \\ de l'Institut d'Histoire de l'Amérique française}

Abonnez-vous à ces Etudes. L'Institut d'Histoire ne publie que des oeuvres d'une valeur scientifique incontestée.

Déja parues :

Iroquoisie, de M. Léo-Paul Desrosiers (\$2.25)

François Bigot, administrateur français, de M. Guy Frégault -2 vol. - $(\$ 4.50)$

Louis Jolliet - Vie et voyages, du Père Jean Delanglez, s.j. (\$2.50)

On peut s'abonner aux Etudes de l'Institut en faisant un dépôt de \$10. au siège social de l'Institut : 261, avenue Bloomfield, Outremont (8), P.Q. 